Proceedings of the Edinburgh Mathematical Society (2006) 49, 309-329 (C)

DOI:10.1017/S0013091504000859 Printed in the United Kingdom

\title{
INVERSE SPECTRAL PROBLEMS FOR STURM-LIOUVILLE OPERATORS WITH SINGULAR POTENTIALS. IV. POTENTIALS IN THE SOBOLEV SPACE SCALE
}

\author{
ROSTYSLAV O. HRYNIV* AND YAROSLAV V. MYKYTYUK \\ Institute for Applied Problems of Mechanics and Mathematics, \\ 3B Naukova St., 79601 Lviv, Ukraine and \\ Lviv National University, 1 Universytetska St., 79602 Lviv, Ukraine \\ (rhryniv@iapmm.lviv.ua; yamykytyuk@yahoo.com)
}

(Received 20 September 2004)

\begin{abstract}
We solve the inverse spectral problems for the class of Sturm-Liouville operators with singular real-valued potentials from the Sobolev space $W_{2}^{s-1}(0,1), s \in[0,1]$. The potential is recovered from two spectra or from one spectrum and the norming constants. Necessary and sufficient conditions for the spectral data to correspond to a potential in $W_{2}^{s-1}(0,1)$ are established.
\end{abstract}

Keywords: inverse spectral problems; Sturm-Liouville operators; singular potentials; Sobolev spaces

2000 Mathematics subject classification: Primary 34A55

Secondary 34B24; 34L05; 34L20

\section{Introduction}

The aim of this paper is to extend the inverse spectral theory of Sturm-Liouville operators on $(0,1)$ to the case of potentials that are distributions from the Sobolev space $W_{2}^{s-1}(0,1), s \in[0,1]$. The classical inverse Sturm-Liouville theory developed in 1950s by Gel'fand, Levitan, Marchenko and Krein $[\mathbf{6}, \mathbf{1 4}, \mathbf{1 9}]$ covers the case $s=1$, and the other extreme case $s=0$ has recently been treated in $[\mathbf{1}, \mathbf{3}, \mathbf{4}, \mathbf{9}, \mathbf{1 1}, \mathbf{2 4}]$ (see also the references therein). Since, however, many singular potentials of interest in quantum mechanics belong to the intermediate spaces between $W_{2}^{0}(0,1)=L_{2}(0,1)$ and $W_{2}^{-1}(0,1)$ (e.g. the Dirac $\delta$-function and the Coulomb $1 / x$-potential are in $W_{2}^{s-1}(0,1)$ for all $\left.s<\frac{1}{2}\right)$, such an extension seems important for applications as it would provide more precise spectral information about the corresponding models.

We start by recalling some of the classical results of the inverse Sturm-Liouville theory. Suppose that $q \in L_{2}(0,1)$ is real valued and that $h_{0}, h_{1} \in \overline{\mathbb{R}}:=\mathbb{R} \cup\{\infty\}$. Denote by $S\left(q, h_{0}, h_{1}\right)$ a Sturm-Liouville operator in $L_{2}(0,1)$ given by the differential expression

$$
l y=-y^{\prime \prime}+q y
$$

* Present address: Institut für Angewandte Mathematik, Abteilung für Wahrscheinlichkeitstheorie und Mathematische Statistik, Wegelerstraße 6, D-53115 Bonn, Germany (rhryniv@wiener.iam.uni-bonn.de). 
and the boundary conditions

$$
y^{\prime}(0)=h_{0} y(0), \quad y^{\prime}(1)=h_{1} y(1),
$$

$h_{0}=\infty$ or $h_{1}=\infty$ corresponding to the Dirichlet boundary condition $y(0)=0$ or $y(1)=$ 0 , respectively. Spectral properties of the operator $S\left(q, h_{0}, h_{1}\right)$ are well understoodnamely, this operator is self-adjoint, bounded below and has a simple discrete spectrum $\lambda_{1}\left(q, h_{0}, h_{1}\right)<\lambda_{2}\left(q, h_{0}, h_{1}\right)<\cdots$ with a limit point at infinity. Moreover, Borg [2] showed (see also a simpler proof by Levinson [15]) that specification of two spectra for two different values of $h_{0}$ determines $q$ uniquely.

Theorem A. Assume that real-valued integrable functions $\tilde{q}$ and $q$ and parameters $h_{0}, h_{0}^{\prime}, h_{1} \in \overline{\mathbb{R}}, h_{0}^{\prime} \neq h_{0}$, are such that $\lambda_{n}\left(\tilde{q}, h_{0}, h_{1}\right)=\lambda_{n}\left(q, h_{0}, h_{1}\right)$ and $\lambda_{n}\left(\tilde{q}, h_{0}^{\prime}, h_{1}\right)=$ $\lambda_{n}\left(q, h_{0}^{\prime}, h_{1}\right)$ for all $n \in \mathbb{N}$. Then $\tilde{q}=q$ a.e. on $(0,1)$.

The classical inverse Sturm-Liouville theory provides the algorithm of reconstruction of $q$ (as well as of the corresponding boundary conditions) from two spectra and also gives necessary and sufficient conditions in order that two given sequences could be spectra of $S\left(q, h_{0}, h_{1}\right)$ and $S\left(q, h_{0}^{\prime}, h_{1}\right)$ for some potential $q$ on $(0,1)$ and some $h_{0}, h_{0}^{\prime}, h_{1} \in \overline{\mathbb{R}}$, $h_{0}^{\prime} \neq h_{0}$. Typically, such conditions require interlacing of these sequences and their proper asymptotics, as in the following theorem of Marchenko [20, Theorem 3.4.1].

Theorem B. Sequences $\lambda_{1}<\lambda_{2}<\cdots$ and $\mu_{1}<\mu_{2}<\cdots$ of real numbers are spectra of the Sturm-Liouville operators $S(q, \infty, \infty)$ and $S(q, 0, \infty)$ with a potential $q \in L_{2}(0,1)$ if and only if the following conditions are satisfied:

(A1) the sequences $\left(\lambda_{n}\right)$ and $\left(\mu_{n}\right)$ interlace, i.e. $\mu_{k}<\lambda_{k}<\mu_{k+1}$ for all $k \in \mathbb{N}$;

(A2) the numbers $\lambda_{n}$ and $\mu_{n}$ have the representations

$$
\lambda_{n}=\left(\pi n+\frac{c}{n}+\frac{\lambda_{n}^{\prime}}{n}\right)^{2}, \quad \mu_{n}=\left(\pi n-\frac{\pi}{2}+\frac{c}{n}+\frac{\mu_{n}^{\prime}}{n}\right)^{2},
$$

for some $c \in \mathbb{R}$ (equal to $\int q$ ) and some $\ell_{2}$-sequences $\left(\lambda_{n}^{\prime}\right)$ and $\left(\mu_{n}^{\prime}\right)$.

Similar results for finite $h_{1}$ and integrable $q$ have been established by Levitan and Gasymov [17] and Krein [14].

In the recent work by Shkalikov and Savchuk (see $[\mathbf{2 5 , 2 6}]$ and the references therein) most of the classical Sturm-Liouville theory has been generalized to the case of distributional potentials in $W_{2}^{-1}(0,1)$. The corresponding self-adjoint operators could be defined by the form sum method; however, such a definition is rather abstract and does not take into account the differential nature of these operators. The explicit construction of $[\mathbf{2 5}, \mathbf{2 6}]$ define the same operators as differential ones; it rests on the regularization by quasi-derivatives and proceeds as follows. Let $q$ be a real-valued distribution from $W_{2}^{-1}(0,1)$ and let $\sigma \in L_{2}(0,1)$ be any of its distributional primitives; then the differential expression (1.1) (understood in the sense of distributions) can be regularized through

$$
l_{\sigma} y:=-\left(y^{\prime}-\sigma y\right)^{\prime}-\sigma y^{\prime}
$$


on the natural domain

$$
\operatorname{dom} l_{\sigma}:=\left\{y \in W_{1}^{1}(0,1) \mid y^{\prime}-\sigma y \in W_{1}^{1}(0,1), l_{\sigma} y \in L_{2}(0,1)\right\} .
$$

We observe that the derivative $y^{\prime}$ of $y \in \operatorname{dom} l_{\sigma}$ need not be continuous, while the quasiderivative $y^{[1]}:=y^{\prime}-\sigma y$ is (absolutely) continuous by construction. It was shown in [25] that the restriction of $l_{\sigma}$ by the boundary conditions

$$
y^{[1]}(0)=h_{0} y(0), \quad y^{[1]}(1)=h_{1} y(1)
$$

with $h_{0}, h_{1} \in \overline{\mathbb{R}}$ determines a self-adjoint operator in $L_{2}(0,1)$ that is bounded below and has simple discrete spectrum. We remark that choosing $\sigma+h$ as a primitive of $q$ and $h_{0}-h, h_{1}-h$ as the new parameters in the boundary conditions (1.4) does not change the operator. If $h_{0}$ is finite and $h=h_{0}$, this makes the Robin boundary condition a 'Neumann' one for a special choice of the primitive $\sigma$, so that there is no distinguished Neumann boundary condition in this case.

To fix the notation, from now on we shall denote by $\sigma$ the primitive of $q$ of zero mean (i.e. such that $\left.\int \sigma=0\right)$ and by $T\left(q, h_{0}, h_{1}\right)$ the Sturm-Liouville operator given by the differential expression $l_{\sigma}$ of (1.3) with this $\sigma$ and the boundary conditions (1.4). Observe that for a regular potential $q \in L_{2}(0,1)$ the operator $T\left(q, h_{0}, h_{1}\right)$ coincides with $S\left(q, h_{0}+\sigma(0), h_{1}+\sigma(1)\right)$, so that, e.g. $T(q,-\sigma(0), \infty)=S(q, 0, \infty)$.

Take some $q \in W_{2}^{-1}(0,1)$ and $h \in \mathbb{R}$ and denote by $\lambda_{1}<\lambda_{2}<\cdots$ and $\mu_{1}<\mu_{2}<\cdots$ the eigenvalues of the operators $T(q, \infty, \infty)$ and $T(q, h, \infty)$, respectively. It is known $[\mathbf{1}$, $\mathbf{1 1}, \mathbf{2 6}]$ that the sequences $\left(\lambda_{n}\right)$ and $\left(\mu_{n}\right)$ satisfy (A1) and the following relaxed version of (A2):

$\left(\mathrm{A} 2^{\prime}\right)$ the numbers $\lambda_{n}$ and $\mu_{n}$ obey the asymptotics

$$
\lambda_{n}=\left(\pi n+\tilde{\lambda}_{n}\right)^{2}, \quad \mu_{n}=\left(\pi n-\frac{1}{2} \pi+\tilde{\mu}_{n}\right)^{2}
$$

with some $\ell_{2}$-sequences $\left(\tilde{\lambda}_{n}\right)$ and $\left(\tilde{\mu}_{n}\right)$.

Conversely, it was shown in [11] that if two sequences $\left(\lambda_{n}\right)$ and $\left(\mu_{n}\right)$ of real numbers satisfy properties (A1) and (A2'), then there exist unique $q \in W_{2}^{-1}(0,1)$ and $h \in \mathbb{R}$ such that $\left(\lambda_{n}\right)$ and $\left(\mu_{n}\right)$ are the eigenvalues of the Sturm-Liouville operators $T(q, \infty, \infty)$ and $T(q, h, \infty)$, respectively. In other words, the inverse spectral problem of recovering the potential from two spectra is uniquely soluble in the class of Sturm-Liouville operators with singular potentials from $W_{2}^{-1}(0,1)$. The papers [9] and [11] give the corresponding reconstruction algorithm and thus extend the classical inverse Sturm-Liouville theory.

We also observe that some inverse spectral problems for a Sturm-Liouville operator in impedance form $a^{-1}\left(a u^{\prime}\right)^{\prime}$, with an impedance function $a \in W_{2}^{1}(0,1)$, were treated in $[\mathbf{1}, \mathbf{3}, \mathbf{4}, \mathbf{2 4}]$. Such an operator is self-adjoint in $L_{2}((0,1) ; a \mathrm{~d} x)$ under suitable boundary conditions and is unitarily similar to $T\left(q, h_{0}, h_{1}\right)$ with $q=(\sqrt{a})^{\prime \prime} / \sqrt{a} \in W_{2}^{-1}(0,1)$ and suitable $h_{0}, h_{1} \in \overline{\mathbb{R}}$.

One of the main aims of the present work is to show that the inverse spectral problem of determining the potential $q$ from the spectra of the operators $T\left(q, h_{0}, h_{1}\right)$ and $T\left(q, h_{0}^{\prime}, h_{1}\right)$, 
$h_{0}^{\prime} \neq h_{0}$, is completely soluble in the class of Sturm-Liouville operators with potentials $q$ from $W_{2}^{s-1}(0,1)$, for every $s \in[0,1]$. For the sake of definiteness, we shall concentrate on the case $h_{0}=h_{1}=\infty$ and $h_{0}^{\prime}=h \in \mathbb{R}$ only, the other cases being analogous. More precisely, we shall formulate necessary and sufficient conditions on sequences $\left(\lambda_{n}\right)$ and $\left(\mu_{n}\right)$ in order that they should be eigenvalues of the Sturm-Liouville operators $T(q, \infty, \infty)$ and $T(q, h, \infty)$, respectively, for some choice of $q$ in $W_{2}^{s-1}(0,1)$ and $h \in \mathbb{R}$.

For an arbitrary intermediate value $s \in(0,1)$, the direct spectral problem was studied in $[\mathbf{1 0}, \mathbf{1 3}, \mathbf{2 6}]$. For instance, it was proved in $[\mathbf{1 0}]$ that the eigenvalue remainders $\tilde{\lambda}_{n}$ and $\tilde{\mu}_{n}$ defined in (1.5) are, respectively, even and odd sine Fourier coefficients of some function from $W_{2}^{s}(0,1)$ (cf. the above-mentioned cases $s=0$ in $\left(\mathrm{A} 2^{\prime}\right)$ and $s=1$ in $(\mathrm{A} 2)$ ). More exactly, the main result from [10] reads as follows.

Theorem C. Assume that $q \in W_{2}^{s-1}(0,1)$ for some $s \in[0,1]$ and that $h \in \mathbb{R}$ and denote by $\lambda_{1}<\lambda_{2}<\cdots$ and $\mu_{1}<\mu_{2}<\cdots$ the eigenvalues of the Sturm-Liouville operators $T(q, \infty, \infty)$ and $T(q, h, \infty)$, respectively. Define $\tilde{\lambda}_{n}$ and $\tilde{\mu}_{n}$ through (1.5); then the function $\sigma^{*}$ given by

$$
\sigma^{*}(x):=2 \sum_{n=1}^{\infty} \tilde{\mu}_{n} \sin [(2 n-1) \pi x]-2 \sum_{n=1}^{\infty} \tilde{\lambda}_{n} \sin (2 \pi n x)
$$

belongs to $W_{2}^{s}(0,1)$. Moreover, $\sigma^{*}-\sigma \in W_{2}^{2 s}(0,1)$, where $\sigma$ is the distributional primitive of $q$ of zero mean.

In this paper we show that the condition $\sigma^{*} \in W_{2}^{s}(0,1)$ is a sufficient addendum to (A1) and $\left(\mathrm{A} 2^{\prime}\right)$ to guarantee that the corresponding potential belongs to $W_{2}^{s-1}(0,1)$. Our main result is as follows.

Theorem 1.1. In order that two sequences $\left(\lambda_{n}\right)$ and $\left(\mu_{n}\right)$ be eigenvalues of the SturmLiouville operators $T(q, \infty, \infty)$ and $T(q, h, \infty)$, respectively, with some $q \in W_{2}^{s-1}(0,1)$, $s \in[0,1]$ and $h \in \mathbb{R}$, it is necessary and sufficient that assumptions (A1), (A2') hold and that the function $\sigma^{*}$ of (1.6) belongs to $W_{2}^{s}(0,1)$.

We remark that the case $s=1$ of this theorem is more general than Theorem B as it allows different constants $c$ in the asymptotics for $\lambda_{n}$ and $\mu_{n}$ in (1.2) at the cost of allowing the Robin boundary condition $y^{\prime}(0)=[\sigma(0)+h] y(0)$ for the second operator. The same $c$ appears if and only if $\sigma^{*}(0)=0$, and the latter condition is equivalent to having $\sigma(0)+h=0[\mathbf{1 0}]$, which results in Marchenko's Theorem B.

As an intermediate step we solve the inverse spectral problem of recovering the potential of a Sturm-Liouville expression from its Dirichlet spectrum $\left(\lambda_{n}\right)$ and the so-called norming constants $\left(\alpha_{n}\right)$. We recall that

$$
\alpha_{n}:=\left(2 \int_{0}^{1}\left|u_{n}(x)\right|^{2} \mathrm{~d} x\right)^{-1},
$$

where $u_{n}$ is an eigenfunction of the operator $T(q, \infty, \infty)$ that corresponds to the eigenvalue $\lambda_{n}$ and satisfies the initial condition $u_{n}^{[1]}(0)=\sqrt{\left|\lambda_{n}\right|}$. Alternatively, we can reduce the inverse spectral problem of reconstructing the potential $q$ and the number $h$ from two 
spectra to that of recovering $q$ and $h$ from the spectrum $\left(\mu_{n}\right)$ of the operator $T(q, h, \infty)$ and the norming constants $\left(\beta_{n}\right)$; the latter are defined as

$$
\beta_{n}:=\left(2 \int_{0}^{1}\left|v_{n}(x)\right|^{2} \mathrm{~d} x\right)^{-1},
$$

where $v_{n}$ is an eigenfunction of the operator $T(q, h, \infty)$ that corresponds to the eigenvalue $\mu_{n}$ and satisfies the initial conditions $v_{n}(0)=1$ and $v_{n}^{[1]}(0)=h$.

In the case $q \in L_{2}(0,1)$ (i.e. for $s=1$ ) the norming constants $\alpha_{n}$ and $\beta_{n}$ have the asymptotics (cf. [22, Theorem 3.4])

$$
\alpha_{n}=1+\frac{\alpha_{n}^{\prime}}{n}, \quad \beta_{n}=1+\frac{\beta_{n}^{\prime}}{n}
$$

for some $\left(\alpha_{n}^{\prime}\right),\left(\beta_{n}^{\prime}\right) \in \ell_{2}$. Gel'fand and Levitan [6] (see also [16, Chapter II.10]) proved that the mapping sending the triple $\left(q, h_{0}, h_{1}\right) \in L_{1}((0,1), \mathbb{R}) \times \mathbb{R}^{2}$ into the spectral data of the operator $S\left(q, h_{0}, h_{1}\right)$ - the sequences of its eigenvalues and norming constants - is oneto-one and presented the reconstruction algorithm. Dahlberg and Trubowitz [5] specified the structure of the set of the spectral data of the Sturm-Liouville operators $S(q, 0, \infty)$ with Neumann-Dirichlet boundary conditions when $q$ runs through $L_{2}((0,1), \mathbb{R})$. In the case of the Dirichlet boundary conditions, Pöschel and Trubowitz $[\mathbf{2 2}]$ proved that such a mapping is a local real analytic isomorphism of $L_{2}((0,1), \mathbb{R})$ and the set of the spectral data, the latter being endowed with a suitable topology.

In the case $q \in W_{2}^{-1}(0,1)$ (i.e. for $\left.s=0\right)$ the norming constants $\alpha_{n}$ of $T(q, \infty, \infty)$ and $\beta_{n}$ of $T(q, h, \infty)$ have the asymptotics [9]

$$
\alpha_{n}=1+\tilde{\alpha}_{n}, \quad \beta_{n}=1+\tilde{\beta}_{n}, \quad\left(\tilde{\alpha}_{n}\right),\left(\tilde{\beta}_{n}\right) \in \ell_{2} .
$$

It was proved in $[\mathbf{9}]$ that any pair of sequences $\left(\mu_{n}\right)$ and $\left(\beta_{n}\right)$ with $\mu_{1}<\mu_{2}<\cdots$ obeying (A2') and positive $\beta_{n}$ satisfying (1.7) form the spectral data of the operator $T(q, h, \infty)$ for unique $q \in L_{2}(0,1)$ and $h \in \mathbb{R}$; moreover, the mapping between the space of the operators $T(q, h, \infty)$ and the set of their spectral data becomes a real analytic isomorphism in suitable topologies. Similar statements hold for the case of Dirichlet boundary conditions, i.e. for the sequences of $\lambda_{n}$ and $\alpha_{n}$.

Our second aim is to specify the above results for the class of potentials in $W_{2}^{s-1}(0,1)$ with $s \in(0,1)$. The asymptotics of $\alpha_{n}$ and $\beta_{n}$ are specified as follows. We introduce the function $\gamma$ via the formula

$$
\gamma(x):=2 \sum_{n=1}^{\infty} \tilde{\beta}_{n} \cos [(2 n-1) \pi x]-2 \sum_{n=1}^{\infty} \tilde{\alpha}_{n} \cos (2 \pi n x)
$$

and also define

$$
\gamma^{*}(x):=-2 x \sigma^{*}(1-x) .
$$

Theorem 1.2. Assume that $\sigma^{*} \in W_{2}^{s}(0,1)$ for some $s \in[0,1]$. Then the function $\gamma$ also belongs to $W_{2}^{s}(0,1)$; moreover, $\gamma-\gamma^{*} \in W_{2}^{2 s}(0,1)$. 
We see that if the primitive $\sigma$ of $q$ belongs to $W_{2}^{s}(0,1)$, then the sequences $\left(-\tilde{\alpha}_{n}\right)$ and $\left(\tilde{\beta}_{n}\right)$ are even and odd cosine Fourier coefficients, respectively, of the function $\gamma \in W_{2}^{s}(0,1)$ given by (1.8). In the reverse direction the claim is that if the even and odd parts of the functions $\sigma^{*}$ and $\gamma$ belong to $W_{2}^{s}(0,1)$, then $\sigma$ is a $W_{2}^{s}$-function (and hence the potential $q$ belongs to $W_{2}^{s-1}(0,1)$ ). More exactly, the following two statements hold true.

Theorem 1.3. Sequences $\lambda_{1}<\lambda_{2}<\cdots$ and $\alpha_{1}, \alpha_{2}, \ldots$ are eigenvalues and norming constants of the Sturm-Liouville operator $T(q, \infty, \infty)$ with a real-valued potential $q \in W_{2}^{s-1}(0,1), s \in[0,1]$, if and only if the following conditions are satisfied:

(B1) the $\lambda_{n}$ have the representation $\lambda_{n}=\left(\pi n+\tilde{\lambda}_{n}\right)^{2}$, where the numbers $\tilde{\lambda}_{n}, n \in \mathbb{N}$, are the even sine Fourier coefficients of some function from $W_{2}^{s}(0,1)$;

(B2) the $\alpha_{n}$ are positive and the numbers $\tilde{\alpha}_{n}:=\alpha_{n}-1, n \in \mathbb{N}$, are the even cosine Fourier coefficients of some function from $W_{2}^{s}(0,1)$.

Theorem 1.4. Sequences $\mu_{1}<\mu_{2}<\cdots$ and $\beta_{1}, \beta_{2}, \ldots$ are eigenvalues and norming constants of the Sturm-Liouville operator $T(q, h, \infty)$ with a real-valued potential $q \in W_{2}^{s-1}(0,1), s \in[0,1]$, and $h \in \mathbb{R}$ if and only if the following conditions are satisfied:

(C1) the $\mu_{n}$ have the representation $\mu_{n}=\pi\left(n-\frac{1}{2}\right)+\tilde{\mu}_{n}$, where the numbers $\tilde{\mu}_{n}, n \in \mathbb{N}$, are the odd sine Fourier coefficients of some function from $W_{2}^{s}(0,1)$;

(C2) the $\beta_{n}$ are positive and the numbers $\tilde{\beta}_{n}:=\beta_{n}-1, n \in \mathbb{N}$, are the odd cosine Fourier coefficients of some function from $W_{2}^{s}(0,1)$.

The organization of the paper is as follows. In $\S 2$ the asymptotics of the norming constants $\alpha_{n}$ and $\beta_{n}$ are established, based on which Theorem 1.2 is proved. The algorithm of solution of the inverse spectral problems under consideration and the proofs of Theorems 1.1, 1.3 and 1.4 are given in $\S 3$. Finally, some necessary facts about the Sobolev spaces $W_{2}^{s}(0,1)$ and Fourier series therein are gathered together in Appendix A.

\section{Asymptotics of the norming constants}

Suppose that $q \in W_{2}^{-1}(0,1)$ is real-valued, that $\sigma \in L_{2}(0,1)$ is its primitive of zero mean, and that $h \in \mathbb{R}$. Throughout the rest of this section, $\lambda_{1}<\lambda_{2}<\cdots$ and $\mu_{1}<\mu_{2}<\cdots$ will stand for the eigenvalues of the Sturm-Liouville operators $T(q, \infty, \infty)$ and $T(q, h, \infty)$, respectively, that were defined in $\S 1$. Observe that $\lambda_{n}+c$ and $\mu_{n}+c, c \in \mathbb{C}$, are the eigenvalues of the operators $T(q+c, \infty, \infty)$ and $T(q+c, h-c / 2, \infty)$, respectively; it therefore suffices to study the case of positive $\lambda_{n}$ and $\mu_{n}$. Recall also that $\lambda_{n}$ and $\mu_{n}$ satisfy conditions (A1) and $\left(\mathrm{A} 2^{\prime}\right)$ of $\S 1$.

We denote by $u_{ \pm}(\cdot, \lambda)$ and $v_{-}(\cdot, \lambda)$ solutions of the equation $l_{\sigma}(u)=\lambda u\left(l_{\sigma}\right.$ being the differential expression of (1.3)) that satisfy the conditions

$$
u_{-}(0, \lambda)=u_{+}(1, \lambda)=v_{-}^{[1]}(0, \lambda)-h=0, \quad u_{-}^{[1]}(0, \lambda)=u_{+}^{[1]}(1, \lambda)=v_{-}(0, \lambda)=1 .
$$


Observe that according to the definition of $l_{\sigma}$ the equation $l_{\sigma}(u)=\lambda u$ is to be regarded as the first-order system

$$
\frac{\mathrm{d}}{\mathrm{d} x}\left(\begin{array}{c}
u \\
u^{[1]}
\end{array}\right)=\left(\begin{array}{cc}
\sigma & 1 \\
-\sigma^{2}-\lambda & -\sigma
\end{array}\right)\left(\begin{array}{c}
u \\
u^{[1]}
\end{array}\right) .
$$

Since the entries of the above matrix are integrable, this system enjoys the standard existence and uniqueness properties; in particular, the solutions $u_{ \pm}(\cdot, \lambda)$ and $v_{-}(\cdot, \lambda)$ are well defined for all $\lambda \in \mathbb{C}$.

Set $\Phi(\lambda):=u_{-}(1, \lambda)$ and $\Psi(\lambda):=u_{+}^{[1]}(0, \lambda)-h u_{+}(0, \lambda)$; then the numbers $\lambda_{n}$ and $\mu_{n}$ are zeros of $\Phi$ and $\Psi$, respectively. $\Phi$ and $\Psi$ are entire functions of order $\frac{1}{2}$ and hence can be represented by their canonical Hadamard products [11], namely,

$$
\Phi(\lambda)=\prod_{n=1}^{\infty} \frac{\lambda_{n}-\lambda}{\pi^{2} n^{2}}, \quad \Psi(\lambda)=\prod_{n=1}^{\infty} \frac{\mu_{n}-\lambda}{\pi^{2}\left(n-\frac{1}{2}\right)^{2}} .
$$

Moreover, it turns out that the norming constants $\alpha_{n}=\left(\sqrt{2 \lambda_{n}}\left\|u_{-}\left(\cdot, \lambda_{n}\right)\right\|\right)^{-2}$ and $\beta_{n}=\left(\sqrt{2}\left\|v_{-}\left(\cdot, \mu_{n}\right)\right\|\right)^{-2}$ can be expressed via the functions $\Phi$ and $\Psi$ only (cf. $\left.[\mathbf{7}, \mathbf{1 1}]\right)$.

Lemma 2.1. The norming constants $\alpha_{n}$ and $\beta_{n}$ satisfy the following equalities:

$$
\alpha_{n}=\frac{\Psi\left(\lambda_{n}\right)}{2 \lambda_{n} \dot{\Phi}\left(\lambda_{n}\right)}, \quad \beta_{n}=-\frac{\Phi\left(\mu_{n}\right)}{2 \dot{\Psi}\left(\mu_{n}\right)} .
$$

Proof. The Green function $G(x, y, \lambda)$ of the operator $T(q, \infty, \infty)$ (i.e. the kernel of the resolvent $\left.(T(q, \infty, \infty)-\lambda)^{-1}\right)$ equals

$$
G(x, y, \lambda)=\sum_{n=1}^{\infty} \frac{2 \alpha_{n} \lambda_{n} u_{-}\left(x, \lambda_{n}\right) u_{-}\left(y, \lambda_{n}\right)}{\lambda_{n}-\lambda} .
$$

On the other hand, we have

$$
G(x, y, \lambda)=\frac{1}{W(\lambda)} \begin{cases}u_{-}(x, \lambda) u_{+}(y, \lambda), & 0 \leqslant x \leqslant y \leqslant 1 \\ u_{-}(y, \lambda) u_{+}(x, \lambda), & 0 \leqslant y \leqslant x \leqslant 1\end{cases}
$$

where $W(\lambda):=u_{+}(x, \lambda) u_{-}^{[1]}(x, \lambda)-u_{-}(x, \lambda) u_{+}^{[1]}(x, \lambda)$ is the Wronskian of $u_{+}$and $u_{-}$. The value of $W(\lambda)$ is independent of $x \in[0,1]$; in particular, taking $x=0$ and $x=1$ yields

$$
W(\lambda) \equiv u_{+}(0, \lambda) \equiv-u_{-}(1, \lambda) \equiv-\Phi(\lambda) .
$$

Equating the two expressions for the Green function and comparing the residues at the poles $\lambda=\lambda_{n}$, we find that

$$
2 \alpha_{n} \lambda_{n} u_{-}\left(y, \lambda_{n}\right)=\frac{u_{+}\left(y, \lambda_{n}\right)}{\dot{\Phi}\left(\lambda_{n}\right)}
$$


Observe that $u_{-}\left(\cdot, \lambda_{n}\right)$ and $u_{+}\left(\cdot, \lambda_{n}\right)$ are collinear and hence

$$
\frac{u_{+}\left(\cdot, \lambda_{n}\right)}{u_{-}\left(\cdot, \lambda_{n}\right)}=\frac{u_{+}^{[1]}\left(\cdot, \lambda_{n}\right)-h u_{+}\left(\cdot, \lambda_{n}\right)}{u_{-}^{[1]}\left(\cdot, \lambda_{n}\right)-h u_{-}\left(\cdot, \lambda_{n}\right)}=u_{+}^{[1]}\left(0, \lambda_{n}\right)-h u_{+}\left(0, \lambda_{n}\right)=\Psi\left(\lambda_{n}\right) ;
$$

combining the above relations, we conclude that

$$
\alpha_{n}=\frac{u_{+}\left(y, \lambda_{n}\right)}{u_{-}\left(y, \lambda_{n}\right)} \frac{1}{2 \lambda_{n} \dot{\Phi}\left(\lambda_{n}\right)}=\frac{\Psi\left(\lambda_{n}\right)}{2 \lambda_{n} \dot{\Phi}\left(\lambda_{n}\right)}
$$

as claimed.

In a similar fashion we equate two expressions for the Green function of the operator $T(q, h, \infty)$, namely,

$$
\sum_{n=1}^{\infty} \frac{2 \beta_{n} v_{-}\left(x, \mu_{n}\right) v_{-}\left(y, \mu_{n}\right)}{\mu_{n}-\lambda} \equiv \frac{1}{W_{1}(\lambda)} \begin{cases}v_{-}(x, \lambda) u_{+}(y, \lambda), & 0 \leqslant x \leqslant y \leqslant 1 \\ v_{-}(y, \lambda) u_{+}(x, \lambda), & 0 \leqslant y \leqslant x \leqslant 1 .\end{cases}
$$

Here $W_{1}$ is the Wronskian of $u_{+}$and $v_{-}$and it is identically equal to $-\Psi$, as follows from the equalities

$$
W_{1}(\lambda):=u_{+}(x, \lambda) v_{-}^{[1]}(x, \lambda)-v_{-}(x, \lambda) u_{+}^{[1]}(x, \lambda)=h u_{+}(0, \lambda)-u_{+}^{[1]}(0, \lambda)=-\Psi(\lambda) .
$$

Therefore, we find that

$$
\beta_{n}=\frac{u_{+}\left(y, \mu_{n}\right)}{2 v_{-}\left(y, \mu_{n}\right) \dot{\Psi}\left(\mu_{n}\right)}=\frac{u_{+}\left(0, \mu_{n}\right)}{2 \dot{\Psi}\left(\mu_{n}\right)}=-\frac{u_{-}\left(1, \mu_{n}\right)}{2 \dot{\Psi}\left(\mu_{n}\right)}=-\frac{\Phi\left(\mu_{n}\right)}{2 \dot{\Psi}\left(\mu_{n}\right)},
$$

where the second equality is obtained by taking $y=0$, while the third one follows from (2.2). The lemma is proved.

In what follows, we shall say that a function $f \in L_{2}(0,1)$ is odd (respectively, even) if $f(1-x) \equiv-f(x)$ (respectively, if $f(1-x) \equiv f(x)$ ). Denote by $L_{2, \mathrm{o}}(0,1)$ and $L_{2, \mathrm{e}}(0,1)$ the subspaces of $L_{2}(0,1)$ consisting of odd and even functions, respectively. We shall denote by $f_{\mathrm{o}}$ and $f_{\mathrm{e}}$, respectively, the odd and even parts of a function $f$; obviously,

$$
f_{\mathrm{o}}(x)=\frac{1}{2}[f(x)-f(1-x)], \quad f_{\mathrm{e}}(x)=\frac{1}{2}[f(x)+f(1-x)] .
$$

Lemma 2.2. The functions $\Phi$ and $\Psi$ admit the integral representations

$$
\begin{aligned}
& \Phi(\lambda)=\frac{\sin \sqrt{\lambda}}{\sqrt{\lambda}}+\int_{0}^{1} \phi(x) \frac{\sin [\sqrt{\lambda}(1-2 x)]}{\sqrt{\lambda}} \mathrm{d} x, \\
& \Psi(\lambda)=\cos \sqrt{\lambda}+\int_{0}^{1} \psi(x) \cos [\sqrt{\lambda}(1-2 x)] \mathrm{d} x,
\end{aligned}
$$

in which $\phi \in L_{2, \mathrm{o}}(0,1)$ and $\psi \in L_{2, \mathrm{e}}(0,1)$. 
Proof. Using the technique of the transformation operators [12], $\Phi$ and $\Psi$ can be shown to admit the integral representations of the form

$$
\begin{aligned}
& \Phi(\lambda)=\frac{\sin \sqrt{\lambda}}{\sqrt{\lambda}}+\int_{0}^{1} \tilde{\phi}(x) \frac{\sin \sqrt{\lambda} x}{\sqrt{\lambda}} \mathrm{d} x \\
& \Psi(\lambda)=\cos \sqrt{\lambda}+\int_{0}^{1} \tilde{\psi}(x) \cos \sqrt{\lambda} x \mathrm{~d} x
\end{aligned}
$$

with some $L_{2}$-functions $\tilde{\phi}$ and $\tilde{\psi}$; see the detailed derivation in [11]. Now we put

$$
\begin{aligned}
\phi(x) & := \begin{cases}\tilde{\phi}(1-2 x) & \text { if } x \in\left[0, \frac{1}{2}\right], \\
-\tilde{\phi}(2 x-1) & \text { if } x \in\left(\frac{1}{2}, 1\right],\end{cases} \\
\psi(x) & := \begin{cases}\tilde{\psi}(1-2 x) & \text { if } x \in\left[0, \frac{1}{2}\right], \\
\tilde{\psi}(2 x-1) & \text { if } x \in\left(\frac{1}{2}, 1\right] .\end{cases}
\end{aligned}
$$

It is easily seen that $\phi \in L_{2, \mathrm{o}}(0,1), \psi \in L_{2, \mathrm{e}}(0,1)$, and that equalities $(2.3)$ hold. The lemma is proved.

The next lemma tells us that the values of $\Phi$ and $\Psi$ at the points $\lambda_{n}$ and $\mu_{n}$ are expressed through sine and cosine Fourier coefficients of some related functions. In the following, $s_{n}(f)$ and $c_{n}(f)$ will stand for, respectively, the $n$th sine and the $n$th cosine Fourier coefficients of a function $f \in L_{2}(0,1)$; see (A 1) for exact formulae. Also, we denote by $M$ the operator of multiplication by $1-2 x$, i.e. $(M f)(x)=(1-2 x) f(x)$ and put $\tilde{\lambda}_{n}:=\sqrt{\lambda_{n}}-\pi n$ and $\tilde{\mu}_{n}:=\sqrt{\mu_{n}}-\pi\left(n-\frac{1}{2}\right), n \in \mathbb{N}$.

Lemma 2.3. For an arbitrary $f \in L_{2}(0,1)$, the following equalities hold:

(1) $\int_{0}^{1} f(x) \sin \left[\sqrt{\lambda_{n}}(1-2 x)\right] \mathrm{d} x=(-1)^{n+1}\left[s_{2 n}(f)-\tilde{\lambda}_{n} c_{2 n}(M f)+\tilde{\lambda}_{n}^{2} s_{2 n}\left(f_{1}\right)\right]$,

(2) $\int_{0}^{1} f(x) \cos \left[\sqrt{\lambda_{n}}(1-2 x)\right] \mathrm{d} x=(-1)^{n}\left[c_{2 n}(f)+\tilde{\lambda}_{n} s_{2 n}(M f)+\tilde{\lambda}_{n}^{2} c_{2 n}\left(f_{2}\right)\right]$

(3) $\int_{0}^{1} f(x) \sin \left[\sqrt{\mu_{n}}(1-2 x)\right] \mathrm{d} x=(-1)^{n+1}\left[c_{2 n-1}(f)+\tilde{\mu}_{n} s_{2 n-1}(M f)+\tilde{\mu}_{n}^{2} c_{2 n-1}\left(f_{3}\right)\right]$,

(4) $\int_{0}^{1} f(x) \cos \left[\sqrt{\mu_{n}}(1-2 x)\right] \mathrm{d} x=(-1)^{n+1}\left[s_{2 n-1}(f)-\tilde{\mu}_{n} c_{2 n-1}(M f)+\tilde{\mu}_{n}^{2} s_{2 n-1}\left(f_{4}\right)\right]$,

where $f_{j}, j=1,2,3,4$, are some functions from $L_{2}(0,1)$.

Proof. We shall prove only part (1) as the other parts are established analogously. Using the equality

$\sin \left[\sqrt{\lambda_{n}}(1-2 x)\right]=(-1)^{n+1} \cos \left[\tilde{\lambda}_{n}(1-2 x)\right] \sin (2 \pi n x)+(-1)^{n} \sin \left[\tilde{\lambda}_{n}(1-2 x)\right] \cos (2 \pi n x)$, 
the asymptotic relations

$$
\sin t=t+O\left(t^{3}\right), \quad \cos t=1-t^{2} / 2+O\left(t^{4}\right), \quad t \rightarrow 0,
$$

and the fact that $\left(\tilde{\lambda}_{n}\right) \in \ell_{2}$, we find that

$$
\int_{0}^{1} f(x) \sin \left[\sqrt{\lambda_{n}}(1-2 x)\right] \mathrm{d} x=(-1)^{n+1}\left[s_{2 n}(f)-\tilde{\lambda}_{n} c_{2 n}(M f)+\tilde{\lambda}_{n}^{2} a_{n}\right]
$$

for some $\ell_{2}$-sequence $\left(a_{n}\right)$. Clearly, there exists a function $f_{1} \in L_{2}(0,1)$ such that $a_{n}=s_{2 n}\left(f_{1}\right)$ for all $n \in \mathbb{N}$ and the proof of part (1) is complete.

Remark 2.4. Put

$$
\begin{array}{ll}
g_{1}:=\sigma^{*} \tilde{*} M f+\sigma^{*} \tilde{*}\left(\sigma^{*} \hat{*} f_{1}\right), & g_{2}:=-\sigma^{*} \hat{*} M f+\sigma^{*} \hat{*}\left(\sigma^{*} \tilde{*} f_{2}\right), \\
g_{3}:=\sigma^{*} \hat{*} M f+\sigma^{*} \hat{*}\left(\sigma^{*} \tilde{*} f_{3}\right), & g_{4}:=-\sigma^{*} \tilde{*} M f+\sigma^{*} *\left(\sigma^{*} \hat{*} f_{4}\right),
\end{array}
$$

where the operations ' $\hat{*}$ ' and ' $\tilde{*}$ ' are introduced in Appendix A. By virtue of Lemma A 2 we can restate equalities (1)-(4) of the previous lemma as follows:

$$
\begin{aligned}
& \left(1^{\prime}\right) \int_{0}^{1} f(x) \sin \left[\sqrt{\lambda_{n}}(1-2 x)\right] \mathrm{d} x=(-1)^{n+1} s_{2 n}\left(f+g_{1}\right), \\
& \left(2^{\prime}\right) \int_{0}^{1} f(x) \cos \left[\sqrt{\lambda_{n}}(1-2 x)\right] \mathrm{d} x=(-1)^{n} c_{2 n}\left(f+g_{2}\right), \\
& \left(3^{\prime}\right) \int_{0}^{1} f(x) \sin \left[\sqrt{\mu_{n}}(1-2 x)\right] \mathrm{d} x=(-1)^{n+1} c_{2 n-1}\left(f+g_{3}\right), \\
& \left(4^{\prime}\right) \int_{0}^{1} f(x) \cos \left[\sqrt{\mu_{n}}(1-2 x)\right] \mathrm{d} x=(-1)^{n+1} s_{2 n-1}\left(f+g_{4}\right) .
\end{aligned}
$$

If the functions $\sigma^{*}$ and $f$ belong to $W_{2}^{s}(0,1)$ for some $s \in[0,1]$, then $M f \in W_{2}^{s}(0,1)$ by Proposition A 1 , and thus Corollary A 4 implies that the above functions $g_{j}, j=1,2,3,4$, belong to $W_{2}^{2 s}(0,1)$.

Using (2.1), the integral representations for $\Phi$ and $\Psi$, and the asymptotics of $\lambda_{n}$ and $\mu_{n}$, we can show that the norming constants $\alpha_{n}$ and $\beta_{n}$ obey the asymptotics $\alpha_{n}=1+\tilde{\alpha}_{n}$ and $\beta_{n}=1+\tilde{\beta}_{n}$ with $\ell_{2}$-sequences $\left(\tilde{\alpha}_{n}\right)$ and $\left(\tilde{\beta}_{n}\right)$. It turns out that if the spectral data $\left(\lambda_{n}\right)$ and $\left(\mu_{n}\right)$ have more precise asymptotics than those of $\left(\mathrm{A} 2^{\prime}\right)$, then the functions $\phi$ and $\psi$ in (2.3) become smoother, and the asymptotics of $\alpha_{n}$ and $\beta_{n}$ refine.

Lemma 2.5. Assume that the numbers $\tilde{\lambda}_{n}$ and $\tilde{\mu}_{n}$ are such that the function $\sigma^{*}$ of (1.6) belongs to $W_{2}^{s}(0,1)$ for some $s \in[0,1]$. Then the functions $\phi$ and $\psi$ in the integral representation (2.3) of $\Phi$ and $\Psi$ have the form

$$
\phi=-\sigma_{\mathrm{o}}^{*}+\phi_{1}, \quad \psi=\sigma_{\mathrm{e}}^{*}+\psi_{1},
$$

where $\phi_{1}$ and $\psi_{1}$ are, respectively, some odd and even functions from $W_{2}^{2 s}(0,1)$. 
Proof. By virtue of Lemma 2.2 the equality $\Phi\left(\lambda_{n}\right)=0$ can be recast as

$$
\sin \sqrt{\lambda_{n}}+\int_{0}^{1} \phi(x) \sin \left[\sqrt{\lambda_{n}}(1-2 x)\right] \mathrm{d} x=0 .
$$

Observe that $\sin \sqrt{\lambda_{n}}=(-1)^{n} \sin \tilde{\lambda}_{n}$ and that $\sin \tilde{\lambda}_{n}=\tilde{\lambda}_{n}+\tilde{\lambda}_{n}^{2} b_{n}$ for some sequence $\left(b_{n}\right)$ from $\ell_{2}$. Combining this observation with Lemma 2.3, we arrive at the relation

$$
\tilde{\lambda}_{n}-s_{2 n}(\phi)+\tilde{\lambda}_{n} c_{2 n}(M \phi)+\tilde{\lambda}_{n}^{2} s_{2 n}(\hat{\phi})=0
$$

for some odd function $\hat{\phi} \in L_{2, \mathrm{o}}(0,1)$. Using Lemma A 2 and recalling that

$$
\tilde{\lambda}_{n}=-s_{2 n}\left(\sigma^{*}\right)=-s_{2 n}\left(\sigma_{\mathrm{o}}^{*}\right),
$$

we conclude that

$$
\phi=-\sigma_{\mathrm{o}}^{*}-\sigma_{\mathrm{o}}^{*} \tilde{*}\left[(M \phi)-\sigma_{\mathrm{o}}^{*} \hat{*} \hat{\phi}\right] .
$$

In particular, $\phi \in W_{2}^{s}(0,1)$ by Corollary $\mathrm{A} 4$, so that the function $(M \phi)-\sigma_{\mathrm{o}}^{*} \hat{*} \hat{\phi}$ belongs to $W_{2}^{s}(0,1)$, and again by Corollary A 4 we get

$$
\phi_{1}:=\phi+\sigma_{\mathrm{o}}^{*}=\sigma_{\mathrm{o}}^{*} \tilde{*}\left[\sigma_{\mathrm{o}}^{*} \hat{*} \hat{\phi}-(M \phi)\right] \in W_{2}^{2 s}(0,1) .
$$

The fact that $\phi_{1}$ is odd is obvious.

In a similar manner, using the relations $\Psi\left(\mu_{n}\right)=0$ and $\sqrt{\mu_{n}}=\pi\left(n-\frac{1}{2}\right)+\tilde{\mu}_{n}$ and Lemmas 2.2 and 2.3 , we find that

$$
\tilde{\mu}_{n}-s_{2 n-1}(\psi)+\tilde{\mu}_{n} c_{2 n-1}(M \psi)+\tilde{\mu}_{n}^{2} s_{2 n-1}(\hat{\psi})=0
$$

for some function $\hat{\psi} \in L_{2, \mathrm{e}}(0,1)$. Replicating the above reasoning, we conclude that $\psi=\sigma_{\mathrm{e}}^{*}+\psi_{1}$ for some even function $\psi_{1}$ from $W_{2}^{2 s}(0,1)$, as claimed. The proof is complete.

Proof of Theorem 1.2. Formulae (2.1) imply that

$$
\tilde{\alpha}_{n}=\alpha_{n}-1=\frac{\Psi\left(\lambda_{n}\right)-2 \lambda_{n} \dot{\Phi}\left(\lambda_{n}\right)}{2 \lambda_{n} \dot{\Phi}\left(\lambda_{n}\right)}, \quad \tilde{\beta}_{n}=\beta_{n}-1=\frac{-\sqrt{\mu_{n}} \Phi\left(\mu_{n}\right)-2 \sqrt{\mu_{n}} \dot{\Psi}\left(\mu_{n}\right)}{2 \sqrt{\mu_{n}} \dot{\Psi}\left(\mu_{n}\right)}
$$

for all $n \in \mathbb{N}$. According to Lemma 2.2 we have

$$
\begin{array}{r}
\Psi\left(\lambda_{n}\right)-2 \lambda_{n} \dot{\Phi}\left(\lambda_{n}\right)=\int_{0}^{1} \theta_{1}(x) \cos \left[\sqrt{\lambda_{n}}(1-2 x)\right] \mathrm{d} x \\
-\sqrt{\mu_{n}} \Phi\left(\mu_{n}\right)-2 \sqrt{\mu_{n}} \dot{\Psi}\left(\mu_{n}\right)=\int_{0}^{1} \theta_{2}(x) \sin \left[\sqrt{\mu_{n}}(1-2 x)\right] \mathrm{d} x
\end{array}
$$

with $\theta_{1}:=\psi-M \phi$ and $\theta_{2}:=-\phi+M \psi$. Furthermore, in view of Lemma 2.3 and Remark 2.4,

$$
\begin{aligned}
& \int_{0}^{1} \theta_{1}(x) \cos \left[\sqrt{\lambda_{n}}(1-2 x)\right] \mathrm{d} x=(-1)^{n} c_{2 n}\left(\theta_{1}+\tilde{\theta}_{1}\right) \\
& \int_{0}^{1} \theta_{2}(x) \sin \left[\sqrt{\mu_{n}}(1-2 x)\right] \mathrm{d} x=(-1)^{n+1} c_{2 n-1}\left(\theta_{2}+\tilde{\theta}_{2}\right)
\end{aligned}
$$

with some functions $\tilde{\theta}_{1}$ and $\tilde{\theta}_{2}$ from $W_{2}^{2 s}(0,1)$. 
It follows from Lemma 2.2 that

$$
\begin{aligned}
2 \lambda_{n} \dot{\Phi}\left(\lambda_{n}\right) & =\cos \sqrt{\lambda_{n}}+\int_{0}^{1}(1-2 x) \phi(x) \cos \left[\sqrt{\lambda_{n}}(1-2 x)\right] \mathrm{d} x, \\
-2 \sqrt{\mu_{n}} \dot{\Psi}\left(\mu_{n}\right) & =\sin \sqrt{\mu_{n}}+\int_{0}^{1}(1-2 x) \psi(x) \sin \left[\sqrt{\mu_{n}}(1-2 x)\right] \mathrm{d} x .
\end{aligned}
$$

We have

$$
\begin{aligned}
\cos \sqrt{\lambda_{n}} & =(-1)^{n} \cos \tilde{\lambda}_{n}=(-1)^{n}\left(1+\tilde{\lambda}_{n} d_{n}\right), \\
\sin \sqrt{\mu_{n}} & =(-1)^{n+1} \cos \tilde{\mu}_{n}=(-1)^{n+1}\left(1+\tilde{\mu}_{n} e_{n}\right)
\end{aligned}
$$

for some $\ell_{2}$-sequences $\left(d_{n}\right)$ and $\left(e_{n}\right)$. Using Lemma 2.3 and Remark 2.4, we now conclude that

$$
(-1)^{n} 2 \lambda_{n} \dot{\Phi}\left(\lambda_{n}\right)=1+c_{2 n}\left(g_{1}\right), \quad(-1)^{n+1} 2 \sqrt{\mu_{n}} \dot{\Psi}\left(\mu_{n}\right)=1+c_{2 n-1}\left(g_{2}\right)
$$

for some functions $g_{1}$ and $g_{2}$ from $W_{2}^{s}(0,1)$. Since $\dot{\Phi}\left(\lambda_{n}\right) \neq 0$ and $\dot{\Psi}\left(\mu_{n}\right) \neq 0$ for all $n \in \mathbb{N}$, Lemma A 5 implies that

$$
\frac{(-1)^{n}}{2 \lambda_{n} \dot{\Phi}\left(\lambda_{n}\right)}=1+c_{2 n}\left(h_{1}\right), \quad \frac{(-1)^{n+1}}{2 \sqrt{\mu_{n}} \dot{\Psi}\left(\mu_{n}\right)}=1+c_{2 n-1}\left(h_{2}\right)
$$

for some functions $h_{1}$ and $h_{2}$ from $W_{2}^{s}(0,1)$.

We now combine the above relations to conclude that

$$
\tilde{\alpha}_{n}=c_{2 n}\left(\theta_{1}+\tilde{\theta}_{1}\right)\left(1+c_{2 n}\left(h_{1}\right)\right), \quad \tilde{\beta}_{n}=c_{2 n-1}\left(\theta_{2}+\tilde{\theta}_{2}\right)\left(1+c_{2 n-1}\left(h_{2}\right)\right) .
$$

It follows that $\gamma=-\theta_{1}+\theta_{2}+\tilde{\theta}$ for some $\tilde{\theta} \in W_{2}^{2 s}(0,1)$. Since

$$
\begin{aligned}
-\theta_{1}+\theta_{2}=(M-I)(\phi+\psi) & =(M-I)\left(\sigma_{\mathrm{e}}^{*}-\sigma_{\mathrm{o}}^{*}\right)+(M-I)\left(\phi_{1}+\psi_{1}\right) \\
& =\gamma^{*}+(M-I)\left(\phi_{1}+\psi_{1}\right)
\end{aligned}
$$

by Lemma 2.5 and $(M-I)\left(\phi_{1}+\psi_{1}\right) \in W_{2}^{2 s}(0,1)$ by Proposition A 1 , we conclude that the function $\gamma-\gamma^{*}$ is in $W_{2}^{2 s}(0,1)$, as required. The theorem is proved.

Observe that Theorems C and 1.2 give necessary parts of Theorems 1.3 and 1.4. Sufficient parts of these theorems constitute the inverse spectral problem and are treated in the next section.

\section{The inverse problem}

We start by recalling briefly the standard method of recovering the Sturm-Liouville operators $T(q, \infty, \infty)$ and $T(q, h, \infty), h \in \mathbb{R}$, from their spectral data-the sequences of eigenvalues and the corresponding norming constants. This method was suggested by Gel'fand and Levitan in [6] for the case of regular (i.e. locally integrable) potentials and was further developed in $[\mathbf{9}]$ to cover singular potentials from $W_{2}^{-1}(0,1)$. 
As in $\S 1$, we denote by $\lambda_{1}<\lambda_{2}<\cdots$ and $\alpha_{1}, \alpha_{2}, \ldots$, respectively, the eigenvalues and the corresponding norming constants of the operator $T(q, \infty, \infty)$ and by $\mu_{1}<\mu_{2}<\cdots$ and $\beta_{1}, \beta_{2}, \ldots$ those of the operator $T(q, h, \infty)$. The functions

$$
\begin{array}{ll}
\omega_{1}(x):=\sum_{n=1}^{\infty}\left[\alpha_{n} \cos \sqrt{\lambda}_{n} x-\cos (\pi n x)\right], & x \in[0,2], \\
\omega_{2}(x):=\sum_{n=1}^{\infty}\left\{\beta_{n} \cos \sqrt{\mu}_{n} x-\cos \left[\pi\left(n-\frac{1}{2}\right) x\right]\right\}, & x \in[0,2],
\end{array}
$$

belong to $L_{2}(0,2)$ as soon as the functions $\sigma^{*}$ of (1.6) and $\gamma$ of (1.8) are in $L_{2}(0,1)$ (cf. Lemmas 3.1 and 3.3 below). We put, for $x, y \in[0,1]$,

$$
f_{j}(x, y):=\omega_{j}(|x-y|)+(-1)^{j} \omega_{j}(x+y), \quad j=1,2,
$$

and introduce an integral operator $F_{j}$ with kernel $f_{j}$; namely, $F_{j}$ acts in $L_{2}(0,1)$ according to the formula

$$
\left(F_{j} u\right)(x):=\int_{0}^{1} f_{j}(x, y) u(y) \mathrm{d} y .
$$

Also, let $K_{1}$ and $K_{2}$ be the transformation operators for $T(q, \infty, \infty)$ and $T(q, h, \infty)$, respectively. Recall that $K_{j}, j=1,2$, is an integral operator with lower-triangular kernel $k_{j}$, i.e. $k_{j}(x, y)=0$ a.e. on the set $\{(x, y) \mid 0 \leqslant x<t \leqslant 1\}$, and thus

$$
\left(K_{j} u\right)(x)=\int_{0}^{x} k_{j}(x, y) u(y) \mathrm{d} y, \quad j=1,2 .
$$

The operator $I+K_{1}$ transforms solutions of the unperturbed equation $l_{0}(u)=\lambda u$ (i.e. corresponding to $\sigma \equiv 0)$ subject to the Dirichlet initial condition $u(0)=0$ into the solutions of the equation $l_{\sigma}(u)=\lambda u$ subject to the Dirichlet initial condition; the operator $I+K_{2}$ does the same for the Robin boundary condition $y^{[1]}(0)=h y(0)$.

Moreover, $f_{j}$ and $k_{j}$ are related through the so-called Gel'fand-Levitan-Marchenko (GLM) equation:

$$
f_{j}(x, y)+k_{j}(x, y)+\int_{0}^{x} k_{j}(x, t) f_{j}(t, y) \mathrm{d} t=0, \quad 0 \leqslant y \leqslant x \leqslant 1 .
$$

It is known $[\mathbf{8}, \mathbf{9}]$ that this GLM equation is naturally related to the problem of factorization of the operator $I+F_{j}$ in a special manner and that (3.3) is uniquely soluble for $k_{j}$ as soon as the operator $I+F_{j}$ is (uniformly) positive. We note that under properties (B1) and (B2) with $s=0$ the operator $I+F_{1}$ is uniformly positive in $L_{2}(0,1)$, and the same is true of $I+F_{2}$ if (C1) and (C2) hold with $s=0$ (see the details in [9]). Hence both GLM equations of interest possess unique solutions. Moreover, up to an additive constant $C_{j}$, the primitive $\sigma$ of the potential $q$ equals

$$
\sigma(x)=(-1)^{j-1} 2 \omega_{j}(2 x)-2 \int_{0}^{x} k_{j}(x, t) f_{j}(t, x) \mathrm{d} t+C_{j} .
$$


In what follows, we shall restrict ourselves to the case of the operator $T(q, \infty, \infty)$; the proofs for the operator $T(q, h, \infty)$ require only minor modifications.

In order to prove the sufficiency part of Theorem 1.3 we need to show first that the function $\omega_{1}$ belongs to $W_{2}^{s}(0,1)$, then establish some properties of the kernel $k_{1}$, and finally use formula (3.4) to prove the inclusion $\sigma \in W_{2}^{s}(0,1)$.

Lemma 3.1. Assume that the numbers $\tilde{\lambda}_{n}$ and $\tilde{\alpha}_{n}>-1$ are such that there exist functions $g$ and $h$ in $W_{2}^{s}(0,1), s \in[0,1]$, with the property that $\tilde{\lambda}_{n}=s_{2 n}(g)$ and $\tilde{\alpha}_{n}=c_{2 n}(h)$. Then the function $\omega_{1}$ belongs to $W_{2}^{s}(0,2)$.

Proof. Observe first that, by the construction of $\sigma^{*}$ and $\gamma$, we have $\sigma_{\mathrm{o}}^{*}=g_{\mathrm{o}}$ and $\gamma_{\mathrm{e}}=h_{\mathrm{e}}$, whence $\sigma_{\mathrm{o}}^{*} \in W_{2}^{s}(0,1)$ and $\gamma_{\mathrm{e}} \in W_{2}^{s}(0,1)$. We write

$$
\begin{aligned}
2 \omega_{1}(2 x) & =2 \sum_{n=1}^{\infty}\left[\left(1+\tilde{\alpha}_{n}\right) \cos \left(2 \pi n x+2 \tilde{\lambda}_{n} x\right)-\cos (2 \pi n x)\right] \\
& =2 \sum_{n=1}^{\infty} \tilde{\alpha}_{n} \cos (2 \pi n x)+2 \sum_{n=1}^{\infty}\left(1+\tilde{\alpha}_{n}\right)\left[\cos \left(2 \tilde{\lambda}_{n} x\right)-1\right] \cos (2 \pi n x) \\
& \quad-2 \sum_{n=1}^{\infty}\left(1+\tilde{\alpha}_{n}\right) \sin \left(2 \tilde{\lambda}_{n} x\right) \sin (2 \pi n x) \\
& =:-\gamma_{\mathrm{e}}(x)+g_{1}(x)-g_{2}(x),
\end{aligned}
$$

so that it remains to prove that the functions $g_{1}$ and $g_{2}$ belong to $W_{2}^{s}(0,1)$.

Justification of the inclusions $g_{1} \in W_{2}^{s}(0,1)$ and $g_{2} \in W_{2}^{s}(0,1)$ is similar, and we shall give it in detail only for the function $g_{1}$. We have

$$
g_{1}(x)=2 \sum_{n=1}^{\infty}\left(1+\tilde{\alpha}_{n}\right) \cos (2 \pi n x) \sum_{k=1}^{\infty}(-1)^{k} \frac{\left(2 \tilde{\lambda}_{n} x\right)^{2 k}}{(2 k) !} .
$$

For $x \in[0,1]$, the estimate

$$
\sum_{k=1}^{\infty} \frac{\left(2 \tilde{\lambda}_{n} x\right)^{2 k}}{(2 k) !} \leqslant \cosh \left(2 \tilde{\lambda}_{n}\right)-1=O\left(\tilde{\lambda}_{n}^{2}\right)
$$

and the inclusion $\left(\tilde{\lambda}_{n}\right) \in \ell_{2}$ imply that the above double series for $g_{1}$ converges uniformly and absolutely. Changing the summation order, we find that

$$
g_{1}(x)=\sum_{k=1}^{\infty} \frac{(-1)^{k} 2^{2 k}}{(2 k) !} x^{2 k} h_{k}(x),
$$

where

$$
h_{k}(x):=2 \sum_{n=1}^{\infty}\left(1+\tilde{\alpha}_{n}\right) \tilde{\lambda}_{n}^{2 k} \cos (2 \pi n x) .
$$

Put $\tau:=\sigma_{\mathrm{o}}^{*} \tilde{*} \sigma_{\mathrm{o}}^{*}$; then by virtue of Lemma A 2 we find that

$$
h_{k}=(\underbrace{\tau \bar{*} \bar{*} \ldots \bar{*} \tau}_{k \text { times }})+\gamma_{\mathrm{e}} \bar{*}(\underbrace{\tau \bar{*} \bar{*} \cdots \bar{*} \tau}_{k \text { times }})
$$


see the definition of ' $\bar{*}$ ' and ' $\tilde{*}$ ' in Appendix A. Corollary A 4 yields the inclusion $h_{k} \in W_{2}^{s}(0,1)$; moreover, there exists a number $C_{1}>0$ such that

$$
\left\|h_{k}\right\|_{s} \leqslant C_{1}\left(1+\left\|\gamma_{\mathrm{e}}\right\|_{0}\right)\left\|\sigma_{\mathrm{o}}^{*}\right\|_{s}^{2 k} .
$$

Denote by $V$ the operator of multiplication by $x$; then $V^{2 k}$ is bounded both in $L_{2}(0,1)$ and in $W_{2}^{1}(0,1)$ and there exists $C_{2} \geqslant 1$ such that, for all $f \in W_{2}^{1}(0,1)$,

$$
\left\|V^{2 k} f\right\|_{0} \leqslant\|f\|_{0}, \quad\left\|V^{2 k} f\right\|_{1} \leqslant C_{2} k\|f\|_{1} .
$$

Interpolating [18] between $W_{2}^{1}(0,1)$ and $L_{2}(0,1)$, we see that $V^{2 k}$ is bounded in every intermediate space $W_{2}^{s}(0,1)$ and $\left\|V^{2 k} f\right\|_{s} \leqslant C_{2} k^{s}\|f\|_{s}$ for all $f \in W_{2}^{s}(0,1)$. Combining the above relations, we conclude that the series in (3.5) converges in $W_{2}^{s}(0,1)$. Henceforth $g_{1} \in W_{2}^{s}(0,1)$, which completes the proof.

Denote by $\mathfrak{A}_{s}$ the set of all integral operators $K$ over $(0,1)$, whose kernels $k$ possess the following properties:

(i) for every $x \in[0,1]$ the functions $k(x, \cdot)$ and $k(\cdot, x)$ belong to $W_{2}^{s}(0,1)$;

(ii) the mappings

$$
[0,1] \ni x \mapsto k(x, \cdot) \in W_{2}^{s}(0,1), \quad[0,1] \ni x \mapsto k(\cdot, x) \in W_{2}^{s}(0,1)
$$

are continuous.

The results of $[\mathbf{2 1}]$ imply the following statement.

Proposition 3.2. Assume that $F$ is an integral operator with kernel $f$ such that $F \in \mathfrak{A}_{s}, s \in\left[0, \frac{1}{2}\right)$, and $I+F>0$. Let $k$ be equal to a solution of the corresponding GLM equation (3.3) in the domain $0 \leqslant y \leqslant x \leqslant 1$ and be zero in the domain $0 \leqslant x<y \leqslant 1$; then an integral operator $K$ with kernel $k$ also belongs to $\mathfrak{A}_{s}$.

Let the assumptions of Lemma 3.1 hold for some $s \in\left[0, \frac{1}{2}\right)$, so that $\omega_{1} \in W_{2}^{s}(0,1)$. Then the operator $F_{1}$ given by (3.1), (3.2) belongs to $\mathfrak{A}_{s}$. Indeed, properties (i) and (ii) of the definition of $\mathfrak{A}_{s}$ for the kernel $f_{1}$ follow from the fact that

(a) the operator $P$ restricting a function on $\mathbb{R}$ onto $(0,1)$ is a bounded mapping from $W_{2}^{s}(\mathbb{R})$ into $W_{2}^{s}(0,1)[\mathbf{1 8}$, Theorem 1.9.1];

(b) the translations $T_{t} f(\cdot):=f(\cdot+t), t \in \mathbb{R}$, form a $C_{0}$-group in $W_{2}^{s}(\mathbb{R})[\mathbf{2 1}]$.

With these preliminaries in hand, we can complete the inverse spectral analysis of Theorems 1.1, 1.3 and 1.4.

Proof of Theorem 1.1. The necessity part of the theorem follows from Theorem C, hence we need to prove only the sufficiency part, i.e. that properties (A1) and (A2') and the inclusion $\sigma^{*} \in W_{2}^{s}(0,1)$ imply that $q \in W_{2}^{s-1}(0,1)$.

Assume therefore that the sequences $\left(\lambda_{n}\right)$ and $\left(\mu_{n}\right)$ satisfy properties (A1) and (A2'). By [11], there exist then a unique function $q \in W_{2}^{-1}(0,1)$ and a unique number $h \in \mathbb{R}$ 
such that $\lambda_{n}$ and $\mu_{n}$ are eigenvalues of the Sturm-Liouville operators $T(q, \infty, \infty)$ and $T(q, h, \infty)$, respectively. It remains to prove that the inclusion $\sigma^{*} \in W_{2}^{s}(0,1)$ yields $\sigma \in W_{2}^{s}(0,1), \sigma$ being the primitive of $q$ of zero mean. We shall consider separately the cases $s \in\left[0, \frac{1}{2}\right), s \in\left[\frac{1}{2}, 1\right)$ and $s=1$.

Case $1\left(s \in\left[\mathbf{0}, \frac{1}{2}\right)\right)$. By Theorem 1.2 the function $\gamma$ belongs to $W_{2}^{s}(0,1)$; hence by Lemma 3.1 the function $\omega_{1}$ is in $W_{2}^{s}(0,1)$ and, as explained above, the integral operator $F_{1}$ falls into the set $\mathfrak{A}_{s}$. It follows from Proposition 3.2 that the solution $k_{1}$ of the GLM equation (3.3) generates an integral operator $K_{1}$ that also belongs to $\mathfrak{A}_{s}$.

In view of formula (3.4) and the inclusion $\omega_{1} \in W_{2}^{s}(0,1)$, the theorem will be proved as soon as we show that the integral $\int_{0}^{x} k_{1}(x, t) f_{1}(t, x) \mathrm{d} t$ defines a function from $W_{2}^{s}(0,1)$. Observe that $k_{1}(x, t)=0$ a.e. for $0 \leqslant x<t \leqslant 1$, so that we can extend the range of integration to $[0,1]$. Hence we put

$$
\eta(x):=\int_{0}^{1} k_{1}(x, t) f_{1}(t, x) \mathrm{d} t
$$

and we will prove that $\eta \in W_{2}^{s}(0,1)$.

Recall [18, Theorem 1.10.2] that one of the equivalent norms in the space $W_{2}^{s}(0,1)$ is given by

$$
\|\eta\|_{s}=\left(\|\eta\|_{0}^{2}+2 \int_{0}^{1} \int_{x}^{1} \frac{|\eta(x)-\eta(y)|^{2}}{(x-y)^{1+2 s}} \mathrm{~d} y \mathrm{~d} x\right)^{1 / 2} .
$$

Now we use the fact that the operators $K_{1}$ and $F_{1}$ belong to $\mathfrak{A}_{s}$. In particular, there exists a constant $C_{1}>0$ such that

$$
\max _{x \in[0,1]}\left(\left\|k_{1}(x, \cdot)\right\|_{s}^{2}+\left\|k_{1}(\cdot, x)\right\|_{s}^{2}+\left\|f_{1}(x, \cdot)\right\|_{s}^{2}+\left\|f_{1}(\cdot, x)\right\|_{s}^{2}\right) \leqslant C_{1},
$$

and we use this inequality to derive the estimates

$$
\|\eta\|_{0}^{2} \leqslant \int_{0}^{1}\left(\int_{0}^{1}\left|k_{1}(x, y)\right|^{2} \mathrm{~d} y \int_{0}^{1}\left|f_{1}(y, x)\right|^{2} \mathrm{~d} y\right) \mathrm{d} x \leqslant C_{1}^{2}
$$

and

$$
\begin{aligned}
|\eta(x)-\eta(y)|^{2} & \leqslant 2\left|\int_{0}^{1}\right| k_{1}(x, t)-k_{1}(y, t)|| f_{1}(t, x)|\mathrm{d} t|^{2} \\
& +2\left|\int_{0}^{1}\right| k_{1}(y, t)|| f_{1}(t, x)-f_{1}(t, y)|\mathrm{d} t|^{2} \\
& \leqslant 2 C_{1}\left(\int_{0}^{1}\left|k_{1}(x, t)-k_{1}(y, t)\right|^{2} \mathrm{~d} t+\int_{0}^{1}\left|f_{1}(t, x)-f_{1}(t, y)\right|^{2} \mathrm{~d} t\right) .
\end{aligned}
$$

It follows that

$$
\|\eta\|_{s}^{2} \leqslant C_{1}^{2}+4 C_{1} \int_{0}^{1}\left[\left\|k_{1}(\cdot, t)\right\|_{s}^{2}+\left\|f_{1}(t, \cdot)\right\|_{s}^{2}\right] \mathrm{d} t \leqslant 5 C_{1}^{2},
$$

so that $\eta \in W_{2}^{s}(0,1)$, and the proof for the case $s \in\left[0, \frac{1}{2}\right)$ is complete. 
Case $2\left(s \in\left[\frac{\mathbf{1}}{\mathbf{2}}, \mathbf{1}\right)\right)$. Case 1 applied to the exponent $\frac{1}{2} s$ gives $\sigma \in W_{2}^{s / 2}(0,1)$, so that $\sigma-\sigma^{*} \in W_{2}^{s}(0,1)$ by Theorem C. Since, by assumption, $\sigma^{*} \in W_{2}^{s}(0,1)$, we have $\sigma \in W_{2}^{s}(0,1)$, as required.

Case $3(s=1)$. We again use the bootstrap method: first, by Case $2, \sigma \in W_{2}^{1 / 2}(0,1)$; then, by Theorem C, $\sigma-\sigma^{*} \in W_{2}^{1}(0,1)$. This inclusion yields $\sigma \in W_{2}^{1}(0,1)$, and the proof is complete.

Proof of Theorem 1.3. If $\sigma \in W_{2}^{s}(0,1)$, then $\sigma^{*} \in W_{2}^{s}(0,1)$ by Theorem $\mathrm{C}$, hence $\gamma \in W_{2}^{s}(0,1)$ by Theorem 1.2. Properties (B1) and (B2) then obviously hold as, by the construction of $\sigma^{*}$ and $\gamma, \tilde{\lambda}_{n}=s_{2 n}\left(-\sigma^{*}\right)$ and $\tilde{\alpha}_{n}=c_{2 n}(-\gamma)$.

Conversely, assume that (B1) and (B2) hold. According to the results of $[\mathbf{9}]$, there exists a unique $q \in W_{2}^{-1}(0,1)$ such that $\lambda_{n}$ and $\alpha_{n}$ are eigenvalues and norming constants of $T(q, \infty, \infty)$. We need to prove that validity of (B1) and (B2) implies that the recovered potential $q$ belongs in fact to $W_{2}^{s-1}(0,1)$ (i.e. that the primitive $\sigma$ of $q$ belongs to $\left.W_{2}^{s}(0,1)\right)$. Indeed, $\left(\mathrm{B} 1^{\prime}\right)$ and $\left(\mathrm{B} 2^{\prime}\right)$ yield $\omega_{1} \in W_{2}^{s}(0,2)$ by Lemma 3.1 , and the proof of Theorem 1.1 uses this inclusion to conclude that $\sigma \in W_{2}^{s}(0,1)$. The proof is complete.

The proof of Theorem 1.4 is completely analogous; the only essential change is that Lemma 3.1 should be replaced with the following, its counterpart (we leave both proofs to the reader).

Lemma 3.3. Assume that the numbers $\tilde{\mu}_{n}$ and $\tilde{\beta}_{n}>-1$ are such that there exist functions $g$ and $h$ in $W_{2}^{s}(0,1)$ with the property that $\tilde{\mu}_{n}=s_{2 n-1}(g)$ and $\tilde{\beta}_{n}=c_{2 n-1}(h)$. Then the function $\omega_{2}$ belongs to $W_{2}^{s}(0,1)$.

Acknowledgements. The authors thank the anonymous referees for their critical remarks and useful suggestions that have led to improvement of the paper. The work was partially supported by the Ukrainian Foundation for Basic Research, grant no. 01.07/00172. R.O.H. gratefully acknowledges the financial support of the Alexander von Humboldt Foundation and thanks the Institute for Applied Mathematics of Bonn University for warm hospitality.

\section{Appendix A. Sobolev spaces $W_{2}^{s}(0,1)$ and the Fourier transform therein}

We recall here some facts about the Sobolev spaces $W_{2}^{s}(0,1)$ and Fourier coefficients of functions from these spaces. For details, we refer the reader to [18, Chapter 1].

By definition, the space $W_{2}^{0}(0,1)$ coincides with $L_{2}(0,1)$ and the norm $\|\cdot\|_{0}$ in $W_{2}^{0}(0,1)$ is just the $L_{2}(0,1)$-norm. The Sobolev space $W_{2}^{2}(0,1)$ consists of all functions $f$ in $L_{2}(0,1)$ whose distributional derivatives $f^{\prime}$ and $f^{\prime \prime}$ also fall in $L_{2}(0,1)$. Being endowed with the norm

$$
\|f\|_{2}:=\left(\|f\|_{0}^{2}+\left\|f^{\prime}\right\|_{0}^{2}+\left\|f^{\prime \prime}\right\|_{0}^{2}\right)^{1 / 2}
$$

$W_{2}^{2}(0,1)$ becomes a Hilbert space.

Now we interpolate [18, Chapter 1.2.1] between $W_{2}^{2}(0,1)$ and $W_{2}^{0}(0,1)$ to get the intermediate spaces $W_{2}^{s}(0,1)$ with norms $\|\cdot\|_{s}$ for $s \in(0,2)$; namely,

$$
W_{2}^{2 t}(0,1):=\left[W_{2}^{2}(0,1), W_{2}^{0}(0,1)\right]_{1-t}, \quad t \in(0,1) .
$$


The norms $\|\cdot\|_{s}$ are non-decreasing with $s \in[0,2]$, i.e. if $s<r$ and $f \in W_{2}^{r}(0,1)$, then $\|f\|_{s} \leqslant\|f\|_{r}$. Since, by construction, the spaces $W_{2}^{s}(0,1)$ form an interpolation scale, the general interpolation theorem [18, Theorem 1.5.1] implies the following interpolation property for operators in these spaces.

Proposition A 1. Assume that an operator $T$ acts boundedly in $W_{2}^{s}(0,1)$ and $W_{2}^{r}(0,1), s<r$. Then $T$ is a bounded operator in $W_{2}^{t s+(1-t) r}(0,1)$ for every $t \in[0,1]$; moreover, $\|T\|_{t s+(1-t) r} \leqslant\|T\|_{s}^{t}\|T\|_{r}^{1-t}$.

Proposition A 1 yields boundedness in every $W_{2}^{s}(0,1), s \in[0,2]$, of the operators $R$ and $V$ given by $R f(x)=f(1-x)$ and $V f(x)=x f(x)$.

For an arbitrary $f \in L_{2}(0,1)$ and an arbitrary $\lambda \in \mathbb{C}$, we put

$$
s_{\lambda}(f):=\int_{0}^{1} f(x) \sin (\pi \lambda x) \mathrm{d} x, \quad c_{\lambda}(f):=\int_{0}^{1} f(x) \cos (\pi \lambda x) \mathrm{d} x .
$$

As usual, ' $*$ ' denotes the convolution operation on $(0,1)$, i.e.

$$
(f * g)(x):=\int_{0}^{x} f(x-t) g(t) \mathrm{d} t .
$$

We shall also introduce the following shorthand notation:

$$
\begin{aligned}
& (f \bar{*} g)(x):=\frac{1}{2}[R(R f * g+f * R g)+f * g+R f * R g], \\
& (f \hat{*} g)(x):=\frac{1}{2}[R(R f * g+f * R g)-f * g-R f * R g], \\
& (f \tilde{*} g)(x):=\frac{1}{2}[R(R f * g-f * R g)+f * g-R f * R g]
\end{aligned}
$$

(where, as earlier, $R$ stands for the reflection operator, $(R f)(x)=f(1-x)$ ). The operations ' $\bar{*}$ ', ' $\hat{*}$ ' and ' $\tilde{*}$ ' play the same role for the sine and cosine Fourier transform on $(0,1)$ as the usual convolution for the Fourier transform on the whole line. Namely, these operations have the following properties.

Lemma A 2. For arbitrary $f, g \in L_{2}(0,1)$ and $\lambda \in \mathbb{C}$ the following equalities hold:

$$
c_{\lambda}(f) c_{\lambda}(g)=c_{\lambda}(f \bar{*} g), \quad s_{\lambda}(f) s_{\lambda}(g)=c_{\lambda}(f \hat{*} g), \quad s_{\lambda}(f) c_{\lambda}(g)=s_{\lambda}(f \tilde{*} g) .
$$

Proof. We shall prove only the first equality since the other two can be treated analogously. We have

$$
2 c_{\lambda}(f) c_{\lambda}(g)=\int_{0}^{1} \int_{0}^{1} f(x) g(t)\{\cos [\pi \lambda(x-t)]+\cos [\pi \lambda(x+t)]\} \mathrm{d} x \mathrm{~d} t,
$$

and simple calculations lead to

$$
\begin{aligned}
\int_{0}^{1} \int_{0}^{1} f(x) g(t) & \cos \pi \lambda(x-t) \mathrm{d} x \mathrm{~d} t \\
= & \int_{0}^{1}\left(\int_{0}^{1-s} f(s+t) g(t) \mathrm{d} t+\int_{0}^{1-s} f(t) g(s+t) \mathrm{d} t\right) \cos (\pi \lambda s) \mathrm{d} s
\end{aligned}
$$




$$
\begin{aligned}
\int_{0}^{1} \int_{0}^{1} f(x) g(t) & \cos [\pi \lambda(x+t)] \mathrm{d} x \mathrm{~d} t \\
& =\int_{0}^{1}\left(\int_{0}^{s} f(s-t) g(t) \mathrm{d} t+\int_{0}^{s} f(1-t) g(1-s+t) \mathrm{d} t\right) \cos \pi \lambda s \mathrm{~d} s
\end{aligned}
$$

Taking into account the relations

$$
\int_{0}^{1-s} f(s+t) g(t) \mathrm{d} t=R(R f * g)(s), \quad \int_{0}^{s} f(1-t) g(1-s+t) \mathrm{d} t=R f * R g
$$

we get $c_{\lambda}(f) c_{\lambda}(g)=c_{\lambda}(f \mp g)$ as stated. The lemma is proved.

It is well known that convolution accumulates smoothness; the precise meaning of this statement is as follows.

Proposition A 3. Assume that $s, t \in[0,1]$ and that $f \in W_{2}^{s}(0,1)$ and $g \in W_{2}^{t}(0,1)$ are arbitrary. Then the function $h:=f * g$ belongs to $W_{2}^{s+t}(0,1)$ and, moreover, there exists $C>0$ independent of $f$ and $g$ such that $\|h\|_{s+t} \leqslant C\|f\|_{s}\|g\|_{t}$.

Proof of this proposition is based on interpolation between the extreme cases $s, t=0,1$, which are dealt with directly.

Combining Proposition A 3 with the fact that the operator $R$ is bounded in the spaces $W_{2}^{s}(0,1)$ for all $s \in[0,1]$, we arrive at the following conclusion.

Corollary A 4. Assume that $s, t \in[0,1]$ and that $f \in W_{2}^{s}(0,1), g \in W_{2}^{t}(0,1)$. Then the functions $f \widetilde{*} g, f \hat{*} g$ and $f \tilde{*} g$ belong to $W_{2}^{s+t}(0,1)$ and, moreover, there exists a number $C>0$ independent of $f$ and $g$ such that

$$
\max \left\{\|f \bar{*} g\|_{s+t},\|f \hat{*} g\|_{s+t},\|f \tilde{*} g\|_{s+t}\right\} \leqslant C\|f\|_{s}\|g\|_{t} .
$$

The following lemma is an analogue of the well-known Wiener lemma.

Lemma A 5. Assume that $f \in W_{2}^{s}(0,1)$, where $s \in[0,1]$. If $1+c_{n}(f) \neq 0$ for all $n \in \mathbb{N}$, then there exists a function $g \in W_{2}^{s}(0,1)$ such that

$$
\left(1+c_{n}(f)\right)^{-1}=1+c_{n}(g), \quad n \in \mathbb{N}
$$

Proof. We start the proof with some auxiliary constructions. Denote by $C$ the operator that acts from $L_{2}(0,1) / \mathbb{C}$ into $\ell_{2}$ according to the formula

$$
C \tilde{f}:=\left(c_{n}(f)\right)_{n \in \mathbb{N}}, \quad f \in \tilde{f} .
$$

This operator is isomorphic; we put

$$
\mathcal{W}^{s}:=\left\{C \tilde{f} \mid \tilde{f} \in W_{2}^{s}(0,1) / \mathbb{C}\right\}
$$

and endow the linear space $\mathcal{W}^{s}$ with the norm

$$
\|x\|_{\mathcal{W}^{s}}:=\left\|C^{-1} x\right\|_{W_{2}^{s}(0,1) / \mathbb{C}}
$$


In view of Lemma A 2 and Corollary A 4 , the element-wise multiplication $(x y)_{n}:=x_{n} y_{n}$ is a continuous operation in $\mathcal{W}^{s}$. We adjoin to $\mathcal{W}^{s}$ the unit element $e$ (with components $e_{n}$ equal to 1) and denote the resulting unital algebra by $\hat{\mathcal{W}}^{s}$. By a well-known result $[\mathbf{2 3}$, Theorem 10.2] one can introduce an equivalent norm in $\hat{\mathcal{W}}^{s}$ under which $\hat{\mathcal{W}}^{s}$ becomes a commutative Banach algebra.

Assume now that the assumptions of the lemma hold and denote by $x$ an element of $\hat{\mathcal{W}}^{s}$ with components $x_{n}:=1+c_{n}(f)$. We shall prove below that $x$ is invertible in $\hat{\mathcal{W}}^{s}$; it then follows that $x^{-1}=e+y$ for some $y \in \mathcal{W}^{s}$, as required.

It is well known [23, Theorem 11.5] that the element $x$ is invertible in the unital Banach algebra $\hat{\mathcal{W}}^{s}$ if and only if $x$ does not belong to any maximal ideal of $\hat{\mathcal{W}}^{s}$. Assume, on the contrary, that there exists a maximal ideal $\mathfrak{m}$ of $\hat{\mathcal{W}}^{s}$ containing $x$. Since $\hat{\mathcal{W}}^{s}$ contains all finite sequences and none of $x_{n}$ vanishes, $\mathfrak{m}$ also contains all finite sequences. Finite sequences form a dense subset of $\mathcal{W}^{s}$ because the set of all trigonometric polynomials in $\cos \pi n x$ is dense in $W_{2}^{s}(0,1) / \mathbb{C}$. Recalling that maximal ideals are closed, we conclude that $\mathcal{W}^{s} \subset \mathfrak{m}$. Next we observe that $\mathcal{W}^{s}$ is a proper subset of $\mathfrak{m}$ (e.g. $x$ belongs to $\left.\mathfrak{m} \backslash \mathcal{W}^{s}\right)$ and that $\mathcal{W}^{s}$ has codimension 1 in $\hat{\mathcal{W}}^{s}$. Henceforth $\mathfrak{m}=\hat{\mathcal{W}}^{s}$, which contradicts our assumption that $\mathfrak{m}$ is a maximal ideal of $\hat{\mathcal{W}}^{s}$. As a result, $x$ is not contained in any maximal ideal of $\hat{\mathcal{W}}^{s}$ and thus is invertible in $\hat{\mathcal{W}}^{s}$. The lemma is proved.

\section{References}

1. L.-E. ANDERSSON, Inverse eigenvalue problems for a Sturm-Liouville equation in impedance form, Inv. Probl. 4 (1988), 929-971.

2. G. Borg, Eine Umkehrung der Sturm-Liouvilleschen Eigenwertaufgabe, Acta Math. 78(1) (1946), 1-96.

3. C. F. Coleman and J. R. McLaughlin, Solution of the inverse spectral problem for an impedance with integrable derivative, I, Commun. Pure Appl. Math. 46 (1993), 145-184.

4. C. F. Coleman and J. R. McLaughlin, Solution of the inverse spectral problem for an impedance with integrable derivative, II, Commun. Pure Appl. Math. 46 (1993), 185-212.

5. B. Dahlberg and E. Trubowitz, The inverse Sturm-Liouville problem, III, Commun. Pure Appl. Math. 37 (1984), 255-267.

6. I. M. Gel'fand and B. M. Levitan, On the determination of a differential equation from its spectral function, Izv. Akad. Nauk SSSR Ser. Mat. 15(4) (1951), 309-360 (in Russian).

7. F. Gesztesy and B. Simon, On the determination of a potential from three spectra, in Differential operators and spectral theory, pp. 85-92, American Mathematical Soceity Translations, Series 2, vol. 189 (American Mathematical Society, Providence, RI, 1999).

8. I. Gohberg AND M. KReIn, Theory of Volterra operators in Hilbert space and its applications (Nauka Publications, Moscow, 1967, in Russian) (English transl.: American Mathematical Society Translations of Mathematical Monographs, vol. 24 (American Mathematical Society, Providence, RI, 1970)).

9. R. HRYNiv AND YA. MYKYTYUK, Inverse spectral problems for Sturm-Liouville operators with singular potentials, Inv. Probl. 19 (2003), 665-684.

10. R. O. HRyniv And Ya. V. Mykytyuk, Eigenvalue asymptotics for Sturm-Liouville operators with singular potentials, submitted, (available at http://arxiv.org/abs/math.SP/ 0407252).

11. R. O. HRyniv and Ya. V. Mykytyuk, Inverse spectral problems for Sturm-Liouville operators with singular potentials, II, Reconstruction by two spectra, in Functional analy- 
sis and its applications (ed. V. Kadets and W. Żelazko), North-Holland Mathematics Studies, vol. 197, pp. 97-114 (North-Holland, Amsterdam, 2004).

12. R. O. HRyniv and YA. V. Mykytyuk, Transformation operators for Sturm-Liouville operators with singular potentials, Math. Phys. Analysis Geom. 7 (2004), 119-149.

13. T. KAPpeler AND C. MÖHR, Estimates for periodic and Dirichlet eigenvalues of the Schrödinger operators with singular potentials, J. Funct. Analysis 186 (2001), 62-91.

14. M. G. Krein, Solution of the inverse Sturm-Liouville problem, Dokl. Akad. Nauk SSSR 76(1) (1951), 21-24 (in Russian).

15. N. Levinson, The inverse Sturm-Liouville problem, Mat. Tidsskr. B 25 (1949), 25-30.

16. B. M. LEvitan, Inverse Sturm-Liouville problems (Nauka Publications, Moscow, 1984, in Russian) (English transl.: VNU Scientific Press, Utrecht, 1987).

17. B. M. LEvitan And M. G. GaSYmov, Determination of a differential equation from two spectra, Usp. Mat. Nauk 19(2) (1964), 3-63 (in Russian).

18. J.-L. LIONS AND E. MAGENES, Non-homogeneous boundary value problems and applications, vol. I (Springer, 1972).

19. V. A. MARChEnKo, Some questions in the theory of second order differential operators, Dokl. Akad. Nauk SSSR 72(3) (1950), 457-460 (in Russian).

20. V. A. MARChenko, Sturm-Liouville operators and their applications (Naukova Dumka Publications, Kiev, 1977, in Russian) (English transl.: Birkhäuser, Basel, 1986).

21. Ya. V. MyкYтyuk, Factorization of Fredholm operators in operator algebras, Mat. Stud. 21(1) (2004), 87-97 (in Ukrainian).

22. J. Pöschel And E. Trubowitz, Inverse spectral theory, Pure and Applied Mathematics, vol. 130 (Academic, 1987).

23. W. Rudin, Functional analysis, 2nd edn (McGraw-Hill, New York, 1991).

24. W. Rundell And P. E. SACKS, The reconstruction of Sturm-Liouville operators, Inv. Probl. 8 (1992), 457-482.

25. A. M. Savchuk and A. A. Shkalikov, Sturm-Liouville operators with singular potentials, Mat. Zametki 66(6) (1999), 897-912 (in Russian).

26. A. M. SAvChuk And A. A. Shkalikov, Sturm-Liouville operators with distributional potentials, Trudy Mosk. Mat. Obsc. 64 (2003), 159-212 (in Russian). 